\title{
Online 4-D CT Estimation for Patient-Specific Respiratory Motion Based on Real-Time Breathing Signals
}

\author{
Tiancheng $\mathrm{He}^{1,2}$, Zhong Xue ${ }^{1, *}$, Weixin $\mathrm{Xie}^{2}$, and Stephen T.C. Wong ${ }^{1}$ \\ ${ }^{1}$ The Center for Bioengineering and Informatics, The Methodist Hospital Research Institute and \\ Department of Radiology, The Methodist Hospital, Weil Cornell Medical College, Houston, TX \\ ${ }^{2}$ Intelligent Information Institute, Shenzhen University, Shenzhen, China \\ zxue@tmhs.org
}

\begin{abstract}
In image-guided lung intervention, the electromagnetic (EM) tracked needle can be visualized in a pre-procedural CT by registering the EM tracking and the CT coordinate systems. However, there exist discrepancies between the static pre-procedural CT and the patient due to respiratory motion. This paper proposes an online 4-D CT estimation approach to patient-specific respiratory motion compensation. First, the motion patterns between 4-D CT data and respiratory signals such as fiducials from a number of patients are trained in a template space after image registration. These motion patterns can be used to estimate the patient-specific serial CTs from a static 3-D CT and the real-time respiratory signals of that patient, who do not generally take 4-D CTs. Specifically, the respiratory lung field motion vectors are projected onto the Kernel Principal Component Analysis (K-PCA) space, and a motion estimation model is constructed to estimate the lung field motion from the fiducial motion using the ridge regression method based on the least squares support vector machine (LS-SVM). The algorithm can be performed onsite prior to the intervention to generate the serial CT images according to the respiratory signals in advance, and the estimated CTs can be visualized in real-time during the intervention. In experiments, we evaluated the algorithm using leave-one-out strategy on 30 4-D CT data, and the results showed that the average errors of the lung field surfaces are $1.63 \mathrm{~mm}$.
\end{abstract}

Keywords: Image-guided intervention, respiratory motion, 4-D CT, K-PCA, least squares support vector machine.

\section{Introduction}

Image-guided intervention has been widely used in different procedures such as lung, liver, and kidney intervention, bronchoscopy, and endovascular interventions. In percutaneous lung intervention, traditional systems use a static pre-procedural CT for guidance and might generate larger errors due to the poor reproducibility of breathholding and the dynamic deformation of lung parenchyma during respiratory cycles. The problem is further multiplexed by the simultaneous deformation of airways, bronchi and vascular structures. Therefore, a precise prediction of the movement of 
the lung or acquiring 4-D CT would be highly desirable for more accurate localization. For percutaneous lung intervention, 4-D CT acquisition is normally not performed in major hospitals. Therefore, it is necessary to simulate the serial CT images from a 3-D CT of that patient based on the real-time tracked signals such as the respiratory belt or the fiducial signals on the chest.

Although complex biomechanical models [1] can be constructed to model the lung motion, image processing is still necessary to adapt these models to a patient. In addition, most biomechanical models need patient-specific 4-D data [2], which means that in a clinical scenario, motion information can only be incorporated if the 4-D images of the patient are additionally acquired. Little work has been presented for building motion models based on the extracted motion fields from 4-D data without considering biomechanical tissue properties. Sundaram et al. [3] first classified the serial images from free-breathing lung MR scans according to the normalized lung capacity and then registered the images with similar capacities to create a dynamic model of average lung deformation. However, this method only constructs the respiratory template dynamics and does not discuss motion modeling and serial image estimation. Motion model adaptation of an inter-subject model using sparse motion information had been recently presented for liver deformation to predict the drift of the exhalation position of corresponding points inside the liver [4]. Daniel A. Low et al. [5, 6] did the work for using 4D-CT lung registration to estimate the 4-D CT lung field motion.

In this paper, we propose a framework for simulating patient-specific serial images based on the 3-D data and the real-time breathing signals of the patient. First, the 4-D CT data from a number of subjects are captured and aligned onto a template space according to their baselines (the first timepoint image), and K-PCA [7] is then applied on the lung field motion vectors derived from the extracted lung field surfaces to construct the nonlinear statistics of lung motion. To establish the relationship between the fiducials' movement and lung surfaces' motion in the template space, the ridge regression method using the least squares support vector machine (LS-SVM) [8] is employed. Then, during the intervention, a 3-D CT and the real-time tracking signal of fiducials and respiratory belts of the patient will be available. Thus, we can use the trained motion estimation model to estimate the lung field motion from the real-time fiducial signals. This motion estimation can be performed in the template space. Finally, the estimated lung field motion can be used to generate serial deformations and serial images for that patient. The estimated serial images can be pre-calculated right after obtaining the intra-procedure $\mathrm{CT}$ and before the intervention in order to visualize them in real-time during the intervention.

In experiments, we used thirty 4-D CT data to construct the motion estimation model and applied the leave-one-out method to validate the algorithm. The results showed that the average differences between the simulated data and the real data (lung field surfaces) are $1.63 \mathrm{~mm}$. Our future work is to simulate the 4-D CT under breath holding and to embed the 4-D CT estimation package in our image-guided intervention system under development [9]. 


\section{Method}

\subsection{The Framework for 4-D CT Estimation}

Fig. 1 illustrates the framework of the proposed 4-D CT estimation algorithm. The pre-processing consists of lung field segmentation, serial image registration for lung motion estimation, and registration of the first timepoint images of different subjects onto a template image. In the training stage, the normalized lung field surface motion vectors and the corresponding fiducial motion vectors of each subject are extracted. Kernel-PCA is then performed on the surface motion vectors to construct the lung motion statistical model and reduce the dimensionality of surface points. Then, a lung motion estimation model is trained using the LS-SVM algorithm to model the relationship between the fiducial signals and the lung motion feature vectors projected on the K-PCA space. In the estimation stage, an intra-procedural 3-D CT and the realtime tracked fiducial signals of a patient are available. The respiratory signals of that patient can be transferred onto the template space in order to use the motion estimation model to estimate the lung motion feature vectors and to reconstruct the lung motion vectors (surface motion vectors) of the patient. Serial deformations can be generated by using the surface motion vectors as constraints in the serial deformation simulator [10]. These serial deformations are finally transformed onto the subject space to generate the serial CTs for online visualization during intervention.

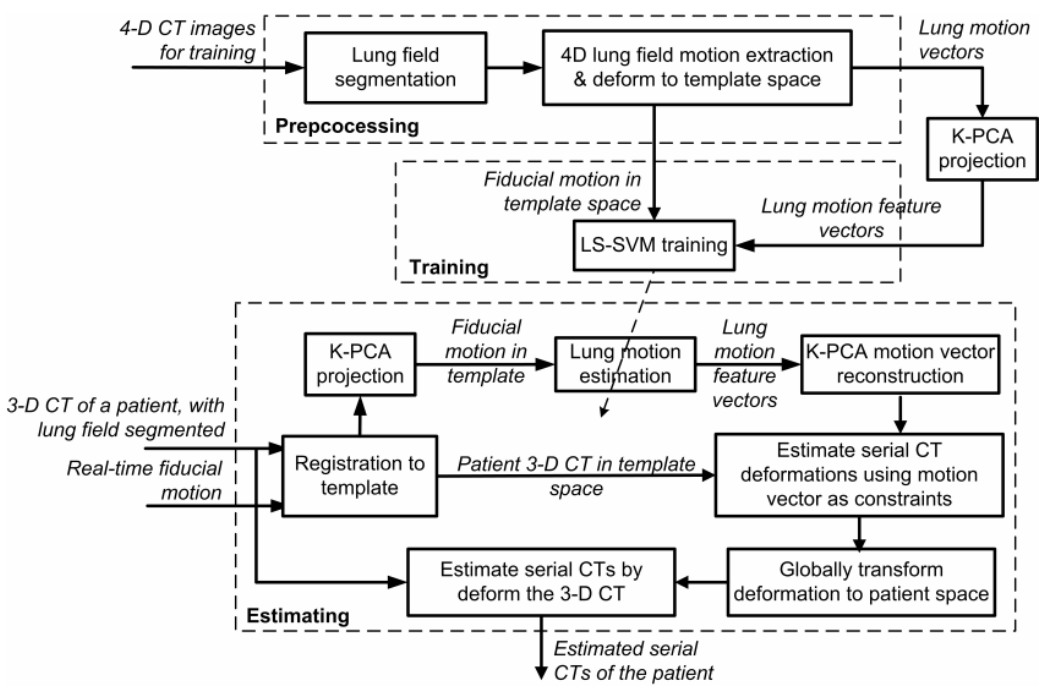

Fig. 1. The framework of the proposed 4-D CT estimation algorithm

\subsection{Motion Field Extraction}

Lung field segmentation is a critical pre-processing step in motion field extraction to limit the motion modeling step within the thorax field and for better visualization. For each subject, we applied a joint segmentation and registration algorithm [11] and extracted the lung field by first removing the background and the cavity areas using 


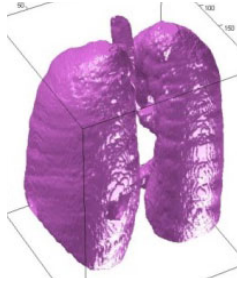

(a)

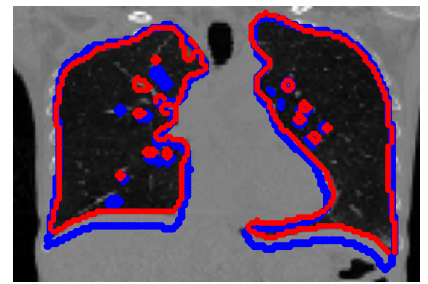

(b)

Fig. 2. An example of the lung field surface and the lung field shape changes

region growing and then performing 3-D morphological operations to clean up the segmented lung field. By registering the baseline image of each subject onto the template space, the surfaces of the lung field from different subjects and their motion vectors can be deformed onto the template space. In our study, serial CT images from thirty patients, each with twelve timepoints were used. For each subject the baseline image is the exhale image and the $7^{\text {th }}$ image is the inhale image, and the $12^{\text {th }}$ image is the exhale image again. Fig. 2(a) shows an example of a lung field extracted, and Fig. 2(b) shows the surfaces extracted from the $7^{\text {th }}$ image before (blue) and after (red) registered to the $1^{\text {st }}$ image (the $1^{\text {st }}$ image is shown as the background). It can be seen that the deformable registration tracked respiratory motion well.

\subsection{Motion Modeling Using Kernel-PCA}

K-PCA is a nonlinear statistical modeling method and can capture the variations of shapes more accurately than PCA. The basic idea of K-PCA is that PCA computed in a high-dimensional implicit mapping function $\varphi(\mathbf{v})$, or the feature space, of the surface motion vector $\mathbf{v}$ can be replaced by a PCA of the kernel matrix. Let $K$ denotes the kernel matrix of $N$ sample surface motion vectors, i.e., $k_{i, j}=k\left(\mathbf{v}_{i}, \mathbf{v}_{j}\right)$, K-PCA can be computed in a closed form by finding the first $M$ eigenvalues $\boldsymbol{v}_{i}$ and eigenvectors $\mathbf{a}_{i}$ of $K$, i.e., $K A=A V$. The corresponding eigenvectors in the feature space can be computed by multiplying the mapping function values of the samples with $A$, and they preserve the variance of data in the feature space. Therefore, given a surface motion vector $\mathbf{v}$, it can be projected onto the K-PCA space as,

$$
\lambda=A^{T}(\mathbf{k}-\overline{\mathbf{k}}),
$$

where $\overline{\mathbf{k}}$ is the mean of the kernel vectors, and $\mathbf{k}$ is the kernel vector of $\mathbf{v}$, i.e., $k_{i}=k\left(\mathbf{v}, \mathbf{v}_{i}\right), i=1, \ldots N$. Because in K-PCA the feature space is induced implicitly, reconstruction of a new vector $\mathbf{v}$ given a feature $\lambda$ is not trivial. Many methods were proposed for the K-PCA reconstruction, and different cost functions could lead to different optimization problems. In this work, we used Kwok \& Tsang's algorithm for reconstruction [12].

\subsection{Motion Prediction Modeling Using LS-SVM}

The goal of motion estimation is to establish the relationship between the lung field surface motion $\mathbf{v}$ (represented by $\lambda$ in the K-PCA space) with the fiducials' motion 
$\mathbf{v}^{(d)}$. Given $N$ training sample-pairs $\left\{\left(\mathbf{v}_{i}^{(d)}, \boldsymbol{\lambda}_{i}\right)\right\}, i=1, \ldots, N$, the relationship between fiducial $\mathbf{v}_{i}^{(d)}$ and lung field motion feature vector $\lambda_{i}$ needs to be established. In this work, we employ the ridge regression method with the LS-SVM model. Given the time series of the motion vectors $\lambda_{i, t}, i=1, \ldots, N ; t=1, \ldots, T$ and those of the fiducial motion vectors $\mathbf{v}_{i, t}^{(d)}$, the goal is to estimate the motion estimation function, i.e., $\boldsymbol{\lambda}(t)=\boldsymbol{\theta}(\mathbf{v}(t))+\mathbf{e}(t)$, where $\mathbf{e}$ is a random process with zero mean and std $\sigma_{e}^{2}$. Because the elements of $\lambda(t)$ are independent each other in the K-PCA space, we can use the LS-SVM model to estimate each element of $\lambda(t)$. Denoting $\lambda$ as one element of $\lambda$ at time $t$, we can estimate it using:

$$
\lambda=\mathbf{w}^{T} \phi\left(\mathbf{v}^{(d)}\right)+b,
$$

where $\phi()$ denotes a potential mapping function. $\mathbf{w}$ is the weighting vector, and $b$ is the shifting vector. The regularized cost function of the LS-SVM is given by [8],

$$
\begin{gathered}
\min _{\mathbf{w . b . e}} \xi(\mathbf{w}, \mathbf{e})=\frac{1}{2} \mathbf{w}^{T} \mathbf{w}+\frac{\gamma}{2} \sum_{i=1}^{N}\left\|e_{i}\right\|^{2} \\
\text { s.t. } \quad \lambda_{i}=\mathbf{w}^{T} \phi\left(\mathbf{v}_{i}^{(d)}\right)+b+e_{i}, i=1, \ldots, N .
\end{gathered}
$$

$\gamma$ is referred to as the regularization constant. This optimization actually corresponds to a ridge regression in feature space. The Lagrangian method is utilized to solve the constrained optimization problem, and hence the new cost function becomes:

$$
\zeta(\mathbf{w}, b, \mathbf{e} ; \boldsymbol{\alpha})=\xi(\mathbf{w}, \mathbf{e})-\sum_{i=1}^{N} \alpha_{i}\left(\mathbf{w}^{T} \phi\left(\mathbf{v}_{i}^{(d)}\right)+b+e_{i}-\lambda_{i}\right),
$$

with $\alpha_{i}$ as the Lagrange multipliers. According to [8], the conditions for optimality are equivalent to the following linear equation:

$$
\left[\begin{array}{cc}
0 & \mathbf{1}_{N}^{T} \\
\mathbf{1}_{N} & \Omega+\gamma^{-1} I_{N}
\end{array}\right]\left[\begin{array}{l}
b \\
\boldsymbol{\alpha}
\end{array}\right]=\left[\begin{array}{l}
0 \\
\Lambda
\end{array}\right],
$$

where $\Lambda=\left[\lambda_{1}, \ldots, \lambda_{N}\right]^{T}$ is the vector formed by the $N$ samples of an element of vector $\lambda_{t}, \mathbf{1}_{N}=[1, \ldots, 1]^{T} \in \mathbf{R}^{N}, \Omega_{i, j}=\Pi\left(\mathbf{v}_{i, t}^{(d)}, \mathbf{v}_{j, t}^{(d)}\right)=\phi\left(\mathbf{v}_{i, t}^{(d)}\right)^{T} \phi\left(\mathbf{v}_{j, t}^{(d)}\right) \forall i, j=1, \ldots, N$ with $\Pi$ as the positive definite kernel function. Notice that because of the kernel trick, the feature mapping $\phi()$ is never defined explicitly, and we only need to define a kernel function $\Pi(\cdot, \cdot)$ of the fiducial vectors. The typical radial basis function (RBF) kernel $\Pi\left(\mathbf{v}_{d}^{i}, \mathbf{v}_{d}^{j}\right)=\exp \left(-\left\|\mathbf{v}_{d}^{i}-\mathbf{v}_{d}^{j}\right\|^{2} / \sigma^{2}\right)$ is used in our study, where $\sigma$ denotes the bandwidth of the kernel. After solving Eq.(5), we get $\boldsymbol{\alpha}$ and $b$, and the element of lung motion feature vector $\lambda$ can be calculated for given fiducial motion vector $\mathbf{v}^{(d)}$ :

$$
\lambda=\sum_{t=1}^{N} \alpha_{t} \Pi\left(\mathbf{v}^{(d)}, \mathbf{v}_{i}^{(d)}\right)+b .
$$

Notice that because different elements of the lung motion feature vector $\lambda$ are independent, all of the elements of $\lambda$ at different timepoints are calculated by this model separately, similar to model the motion according to different lung capacity. 


\section{Results}

We used thirty 4-D CT datasets from 30 different patients in the experiments. Twelve 3-D images were acquired for each patient, and the images were aligned so that the first and the last images were exhale data and the $7^{\text {th }}$ data was the inhale data. All the images have an in-plane resolution of $0.98 \times 0.98 \mathrm{~mm}$ and a slice thickness of $1.5 \mathrm{~mm}$. To ensure that we can get a consistent lung field surface representation, one image was randomly selected as the template, and its lung field surface was constructed first. Then, we applied the image segmentation and registration program to deform this lung field surface onto all the other images. In this way, we can obtain the lung field surface correspondences across different subjects and different timepoints to ensure the surfaces have the same trajectory. Using the same strategy, nine artificial fiducials were automatically put on the surface of the chest/belly of each CT image. In our system the EM-guidance is used to track the interventional needle, thus it is valuable to show the feasibility of the motion estimation using EM-tracked fiducials. We used the leaveone-out method to validate the proposed algorithm. Each time the baseline images from twenty eight subjects were registered onto the template image for training the lung motion estimation model. Then, the baseline image of the left-out subject and the fiducal movement signals of this subject were used to estimate the serial CT images.

Without loss of generality, suppose the baseline image of the patient to be tested is $I_{1}^{(p)}$, we can first register it onto the template image $T$ using deformable registration $\Phi_{T-P}: T \rightarrow I_{1}^{(p)}$, and $\Phi_{T-P}=\{G, \mathbf{f}\}$ consists of both global $G$ and deformable $\mathbf{f}$ components of the registration. The corresponding lung field surface of the patient $v_{1}^{(p)}$ can also be aligned onto the template as $v_{1}$. Similarly, the fiducial movement $v_{t}^{(d, p)}$ can be aligned onto the template space, denoted as $v_{t}^{(d)}$. Notice global transformation $G$ needs to be applied to the fiducial motion because we are dealing with different spaces. We can then use Eq. (6) to estimate the serial lung motion feature vectors and reconstruct the lung field motion from K-PCA space to the template image space, denoted as $v_{t}, t=2, \ldots, T$. A lung field motion vector-constrained deformation simulation method is applied to generate the serial deformation fields [13]. Finally, the deformations are transformed onto the subject space using $\Phi_{T-P}$. The values of these deformation vectors are subject to the global transformation $G$ also.

The errors between the estimated lung field surface and the actual surface at each time point as well as the volumes of the lung fields were used to evaluate the accuracy of the estimation. The procedure was iterated 28 times with one subject left out each time after selecting one image as the template. The following equation was used to calculate the prediction errors for lung field surfaces:

$$
\Delta_{i}=\frac{1}{28} \sum_{28 \text { tests }} \operatorname{dist}(\mathbf{v}, \hat{\mathbf{v}}), \text { subject } i \text { is left out. }
$$

The distance between two surfaces is defined as the average of distances from all the points in one surface to the other surface [14]. Another quantitative measure is the volume of the lung field. Because the lung fields from both estimated CT and the original CT images are available, we simply calculated the lung volumes and compare whether they are close. The algorithm was implemented on an HP workstation with 4 Inter 2.5 GHz dual core Quad Q9300 processors and 4 GB RAM. The runtime for 
training the 4D model using 30 samples was around 1 hour, and the runtime for testing each case was less than 1 minute.

Fig. 3 illustrates some examples of the results. From the second row we can see that the estimated lung field and the actual lung field match very well, and this can also be seen from the plots of the lung field volumes in the third row. In Table 1, we list the average lung field estimation errors for 8 experiments, and each result is evaluated on the left-out subject image. For the 8 tests, the maximum amount of lung motion (on the lung field surface) is $10.20 \mathrm{~cm}$, the minimum one is $0.048 \mathrm{~cm}$, and the average lung motion is $4.78 \mathrm{~cm}$. It can be seen that the average errors over the serial images are between $1.22 \mathrm{~mm}$ and $2.18 \mathrm{~mm}$ with an average of $1.63 \mathrm{~mm}$. If we look at the errors at different timepoints in detail, the largest errors happened at timepoint 7 , which is the inhale stage due to relatively larger movement. Overall, an acceptable range of errors were obtained for predicting the lung motion. Our future work will focus on determining and tracking the location of the lung tumor.

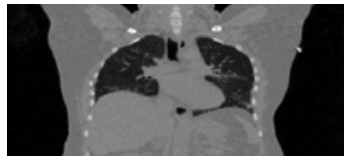

(a1)

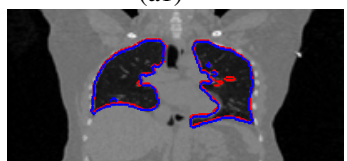

(a2)

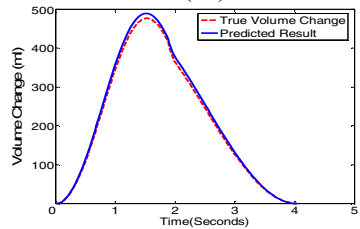

(a3)

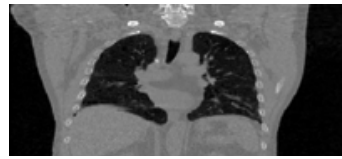

(b1)

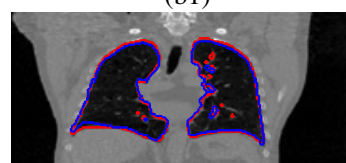

(b2)

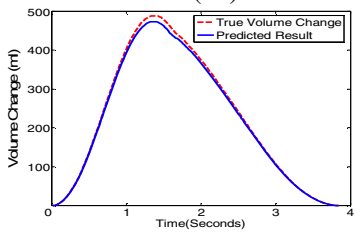

(b3)

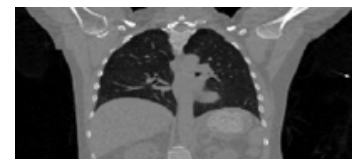

(c1)

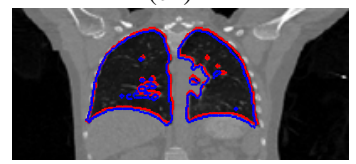

(c2)

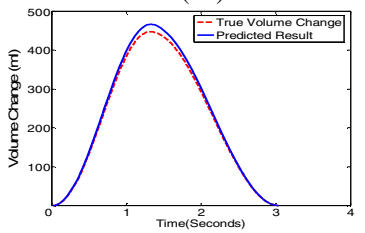

(c3)

Fig. 3. Examples of the motion estimation results. Row 1: the exhale images; Row 2: the corresponding inhale images, blue contours denote the predicted lung field, and red contours are the actual lung field position; Row 3: predicted and actual changes of lung volumes.

Table 1. Average errors for lung motion estimation using leave-one-out validation (unit in $\mathrm{mm}$ )

\begin{tabular}{ccccccccccccr}
\hline Time & T2 & T3 & T4 & T5 & T6 & T7 & T8 & T9 & T10 & T11 & T12 & Mean \\
\hline Pat. 1 & 1.20 & 1.39 & 1.61 & 1.64 & 2.69 & 3.49 & 2.75 & 2.75 & 2.88 & 1.94 & 1.63 & 2.18 \\
Pat. 2 & 0.98 & 1.14 & 1.31 & 1.34 & 2.20 & 2.85 & 2.25 & 2.25 & 2.36 & 1.59 & 1.33 & 1.78 \\
Pat. 3 & 1.08 & 1.25 & 1.44 & 1.47 & 2.42 & 3.14 & 2.47 & 2.48 & 2.59 & 1.75 & 1.46 & 1.96 \\
Pat. 4 & 0.83 & 0.96 & 1.11 & 1.13 & 1.86 & 2.42 & 1.90 & 1.91 & 1.99 & 1.34 & 1.13 & 1.51 \\
Pat. 5 & 0.90 & 1.04 & 1.20 & 1.23 & 2.01 & 2.62 & 2.06 & 2.06 & 2.16 & 1.46 & 1.22 & 1.63 \\
Pat. 6 & 0.77 & 0.89 & 1.03 & 1.05 & 1.73 & 2.24 & 1.77 & 1.77 & 1.85 & 1.25 & 1.05 & 1.40 \\
Pat. 7 & 0.67 & 0.78 & 0.90 & 0.92 & 1.51 & 1.96 & 1.54 & 1.55 & 1.62 & 1.09 & 0.92 & 1.22 \\
Pat. 8 & 0.72 & 0.83 & 0.96 & 0.98 & 1.61 & 2.09 & 1.65 & 1.65 & 1.73 & 1.16 & 0.98 & 1.31 \\
\hline
\end{tabular}

\section{Conclusion}

We proposed an online 4-D CT image estimation approach to patient-specific respiratory motion compensation. The idea is that a motion estimation model is first trained 
using a number of 4-D CTs from different subjects. Then, this model can be used to simulate serial CTs if a 3-D image and the real-time tracked fiducial signals of a patient are given. Leave-one-out validation results from 30 4-D CT data showed the accuracy of the proposed algorithm. Our future work includes simulating 4-D CT under breath holding and implementing the algorithm in image-guided intervention.

\section{Acknowledgement}

The authors would like to thank Dr. Daniel Low and Dr. Wei Lu from Washington University in ST. Louis for providing the 4-D datasets.

\section{References}

1. McClelland, J.R., Blackall, J.M., Tarte, S., et al.: A Continuous 4D Motion Model from Multiple Respiratory Cycles for Use in Lung Radiotherapy. Medical Physics 33, 3348 3358 (2006)

2. Zhang, Q., Pevsner, A., Hertanto, A., Hu, Y.C., Rosenzweig, K.E., Ling, C.C., Mageras, G.S.: A Patient-Specific Respiratory Model of Anatomical Motion for Radiation Treatment Planning. Medical Physics 32, 4772-4782 (2007)

3. Sundaram, T.A., Avants, B.B., Gee, J.C.: A Dynamic Model of Average Lung Deformation Using Capacity-Based Reparameterization and Shape Averaging of Lung MR Images. In: Barillot, C., Haynor, D.R., Hellier, P. (eds.) MICCAI 2004. LNCS, vol. 3217, pp. 1000-1007. Springer, Heidelberg (2004)

4. Von Siebenthal, M., Szkely, G., Lomax, A., Cattin, P.: Inter-Subject Modeling of Liver Deformation During Radiation Therapy. In: Ayache, N., Ourselin, S., Maeder, A. (eds.) MICCAI 2007, Part I. LNCS, vol. 4791, pp. 659-666. Springer, Heidelberg (2007)

5. Lu, W., Song, J.H., Christensen, G.E., Parikh, P.J., Zhao, T., Hubenschmidt, J.P., Bradley, J.D., Low, D.A.: Evaluating Lung Motion Variations in Repeated 4D CT Studies Using Inverse Consistent Image Registration. International Journal of Radiation Oncology Biology Physics 66, S606-S607 (2006)

6. Yang, D.S., Lu, W., Low, D.A., Deasy, J.O., Hope, A.J., El Naqa, I.: 4D-CT Motion Estimation Using Deformable Image Registration and 5D Respiratory Motion Modeling. Medical Physics 35, 4577-4590 (2008)

7. Kim, K.I., Franz, M.O., Scholkopf, B.: Iterative Kernel Principal Component Analysis for Image Modeling. IEEE Trans. on Patt. Analy. and Mach. Intell. 27, 1351-1366 (2005)

8. An, S.J., Liu, W.Q., Venkatesh, S.: Fast Cross-Validation Algorithms for Least Squares Support Vector Machine and Kernel Ridge Regression. Pattern Recognition 40, 2154-2162 (2007)

9. He, T., Xue, Z., Wong, K., Valdivia y Alvarado, M., Zhang, Y., Xie, W., Wong, S.T.C.: Minimally Invasive Multimodality Image-Guided (MIMIG) Molecular Imaging System for Peripharal Lung Cancer Intervention and Diagnosis. In: Navab, N., Jannin, P. (eds.) IPCAI 2010. LNCS, vol. 6135, Springer, Heidelberg (2010)

10. Xue, Z., Shen, D.G., Davatzikos, C.: CLASSIC: Consistent Longitudinal Alignment and Segmentation for Serial Image Computing. Neuroimage 30, 388-399 (2006)

11. Xue, Z., Wong, K., Wong, S.T.C.: Joint Registration and Segmentation of Serial Lung CT Images for Image-Guided Lung Cancer Diagnosis and Therapy. Computerized Medical Imaging and Graphics 34, 55-60 (2010)

12. Kwok, J.T.Y., Tsang, I.W.H.: The Pre-Image Problem in Kernel Methods. IEEE Transactions on Neural Networks 15, 1517-1525 (2004)

13. Xue, Z., Shen, D., Karacali, B., Stern, J., Rottenberg, D., Davatzikos, C.: Simulating Deformations of MR Brain Images for Validation of Atlas-Based Segmentation and Registration Algorithms. Neuroimage 33, 855-866 (2006)

14. Gerig, G., Jomier, M., Chakos, M.: Valmet: A New Validation Tool for Assessing and Improving 3D Object Segmentation. In: Niessen, W.J., Viergever, M.A. (eds.) MICCAI 2001. LNCS, vol. 2208, pp. 516-528. Springer, Heidelberg (2001) 